\title{
Generalized Supersymmetric Perturbation Theory
}

\author{
B. Gönül \\ Department of Engineering Physics, University of Gaziantep, \\ 27310 Gaziantep-Türkiye
}

\begin{abstract}
Using the basic ingredient of supersymmetry, we develop a simple alternative approach to perturbation theory in one-dimensional non-relativistic quantum mechanics. The formulae for the energy shifts and wave functions do not involve tedious calculations which appear in the available perturbation theories. The model applicable in the same form to both the ground state and excited bound states, unlike the recently introduced supersymmetric perturbation technique which, together with other approaches based on logarithmic perturbation theory, are involved within the more general framework of the present formalism.
\end{abstract}

\section{Introduction}

An exact solution of the Schrödinger equation exists only for a few idealized problems; normally it has to be solved using an approximation method such as the perturbation theory (PT), which constitutes one of the most powerful tools available in the study of quantum mechanics in the atoms and molecules. PT is applied to those cases in which the real system can be described by a small change in an exactly solvable idealized system. In this form we can describe a great number of problems encountered especially in atomic physics, in which the nucleus provides the strong central potential for the electrons; further interactions of less strength are described by the perturbation. Examples of these additional interactions are: the magnetic interaction (spin-orbit coupling), the electrostatic repulsion of electrons and the influence of external fields. But in spite of widespread application of this theory, its basic analytical properties are poorly understood. One of our objectives in this paper is to illustrate selected important aspects of the perturbation theory within the frame of supersymmetric quantum mechanics.

Performing explicit calculations in non-relativistic quantum mechanics using the familiar Rayleigh-Schrödinger perturbation expansion is rendered difficult by 
the presence of summations over all intermediate unperturbed eigenstates. Alternative perturbation procedures have been proposed to avoid this difficulty, notably the logaritmic perturbation theory (LPT) [1]-[4] and the Dalgarno-Lewis technique [5]-[8]. The virtue of LPT is its avoidance of the cumbersome summation over states for second- and higher-order corrections in Rayleigh-Schrödinger perturbation theory. Unfortunately, it has problems of its own in calculating corrections to excited states, owing to presence of nodes in the wave functions. Various schemes have been proposed to circumvent the resulting singularities $[4,9,10]$.

Such is the status of LPT after over 20 years of active development. Meanwhile, supersymmetric quantum mechanics (SSQM) [11, 12] has developed immensely since the first models were introduced [13, 14]. Several approximation methods using SSQM formalism have been developed, including the supersymmetric perturbation theory (SSPT) of Cooper and Roy [15]. Recently, Lee [16] has shown that SSPT and LPT are entirely equivalent and fortuitously, each turns out to resolve difficulties encountered in the other. Namely, LPT formulas for energy corrections obviate tedious procedures in the SSQM method, while the use of SSQM partner potentials with virtually identical bound state spectra solves difficulties with excited states encountered in LPT. Although the iterative procedure in SSPT may not actually reduce the calculational workload, it does cast the calculations into a physically-motivated, visualizable framework.

In this letter, starting from the first priciples, we develop a more economical scheme which yields simple but closed perturbation theory formulae leading to the Riccati equation from which one can actually obtain all the perturbation corrections to both energy level shifts and wave functions for all states, unlike the other models mentioned above. In the application of the present method to the $n^{\text {th }}$ excited state, one requires knowledge of the unperturbed eigenfunction $\chi_{n}(r)$ but no knowledge of the other eigenvalues or eigenfunctions is necessary. The procedure introduced here does not involve either tedious explicit factoring out of the zeros of $\chi_{n}(r)[1,2]$ or introduction of ghost states [4] as were the cases encountered for applying LPT to excited states. Since, the present model offers the explicit expressions for the energy corrections, which are absent in the original SSPT while the treatment of Lee [16] for such calculations has mathematical complexity, and provides a clean route to the excited states, which are cumbersome to analyze in both LPT and SSPT, our results can be thought of as a generalization of logarithmic and supersymmetric based perturbation theories. This is the another objective in the present work.

In the following section we introduce the model and discuss briefly the phyiscs behind the formulation. In Section 3, some applications are given and the power of the present technique is illustrated when compared to the calculation technique of other theories considering the whole of states. Some concluding remarks and summary of the work are drawn in the last section. 


\section{Formulation}

The goal in SSQM is to solve the Riccati equation,

$$
W^{2}(r)-\frac{\hbar}{\sqrt{2 m}} W^{\prime}(r)=V(r)-E_{0}
$$

where $V(r)$ is the potential of interest and $E_{0}$ is the corresponding ground state energy. If we find $W(r)$, the so called superpotential, we have of course found the ground state wave function via,

$$
\psi_{0}(r)=N \exp \left[-\frac{\sqrt{2 m}}{\hbar} \int^{r} W(z) d z\right],
$$

where $N$ is the normalization constant. If $V(r)$ is a shape invariant potential, we can in fact obtain the entire spectrum of bound state energies and wave functions via ladder operators [11]. Through the article, this basic ingredient of SSQM given by (1) and (2) will be extended and used for the treatment of excited states.

Now, suppose that we are interested in a potential for which we do not know $W(r)$ exactly, and the corresponding Hamiltonian is not factorizable but almost factorizable. More specifically, we assume that $V(r)$ differs by a small amount from a potential $V_{0}(r)$ plus angular momentum barrier if any, for which one solves the Riccati equation explicitly. For the consideration of spherically symmetric potentials, the corresponding Schrödinger equation for the radial wave function has the form

$$
\frac{\hbar^{2}}{2 m} \frac{\psi_{n}^{\prime \prime}(r)}{\psi_{n}(r)}=\left[V(r)-E_{n}\right] \quad, \quad V(r)=\left[V_{0}(r)+\frac{\hbar^{2}}{2 m} \frac{\ell(\ell+1)}{r^{2}}\right]+\Delta V(r),
$$

where $\Delta V$ is a perturbing potential. Let us write the wave function $\psi_{n}$ as

$$
\psi_{n}(r)=\chi_{n}(r) \phi_{n}(r)
$$

in which $\chi_{n}$ is the known normalized eigenfunction of the unperturbed Schrödinger equation whereas $\phi_{n}$ is a moderating function corresponding to the perturbing potential. Substituting (4) into (3) yields

$$
\frac{\hbar^{2}}{2 m}\left(\frac{\chi_{n}^{\prime \prime}}{\chi_{n}}+\frac{\phi_{n}^{\prime \prime}}{\phi_{n}}+2 \frac{\chi_{n}^{\prime}}{\chi_{n}} \frac{\phi_{n}^{\prime}}{\phi_{n}}\right)=V-E_{n} .
$$

Instead of setting the functions $\chi_{n}$ and $\phi_{n}$, we will set their logarithmic derivatives using the spirit of Eqs. (1) and (2);

$$
W_{n}=-\frac{\hbar}{\sqrt{2 m}} \frac{\chi_{n}^{\prime}}{\chi_{n}}, \Delta W_{n}=-\frac{\hbar}{\sqrt{2 m}} \frac{\phi_{n}^{\prime}}{\phi_{n}}
$$


which leads to

$$
\frac{\hbar^{2}}{2 m} \frac{\chi_{n}^{\prime \prime}}{\chi_{n}}=W_{n}^{2}-\frac{\hbar}{\sqrt{2 m}} W_{n}^{\prime}=\left[V_{0}(r)+\frac{\hbar^{2}}{2 m} \frac{\ell(\ell+1)}{r^{2}}\right]-\epsilon_{n},
$$

where $\epsilon_{n}$ is the eigenvalue of the unperturbed and exactly solvable potential, and

$$
\frac{\hbar^{2}}{2 m}\left(\frac{\phi_{n}^{\prime \prime}}{\phi_{n}}+2 \frac{\chi_{n}^{\prime}}{\chi_{n}} \frac{\phi_{n}^{\prime}}{\phi_{n}}\right)=\Delta W_{n}^{2}-\frac{\hbar}{\sqrt{2 m}} \Delta W_{n}^{\prime}+2 W_{n} \Delta W_{n}=\Delta V(r)-\Delta \epsilon_{n},
$$

in which $\Delta \epsilon_{n}$ is the eigenvalue for the perturbed potential, and $E_{n}=\epsilon_{n}+\Delta \epsilon_{n}$. Then, Eq. (5), and subsequently Eq. (3), reduces to

$$
\left(W_{n}+\Delta W_{n}\right)^{2}-\frac{\hbar}{\sqrt{2 m}}\left(W_{n}+\Delta W_{n}\right)^{\prime}=V-E_{n},
$$

which is similar to Eq. (1). In priciple as one knows explicitly the solution of Eq. (7), namely the whole spectrum and corresponding eigenfunctions of the unperturbed interaction potential, the goal here is to solve only Eq. (8), which is the main result of this letter, leading to the solution of Eqs. (3) and (9).

Eq. (8) is a closed analytical form in comparing to lengthy SSPT and LPT expressions, in particular for the excited states. In this respect, the present formulation has a more general form than the available perturbation theories. Though this point will be clear in the next section through the applications, it would be convenient at this stage to clarify how Eq. (8) involves in a compact form the supersymmetric and logarithmic perturbation theory expressions. As the equivalance of SSPT to LPT has already been clarifed [16], we consider here only the framework of SSPT and show that SSPT is a subset of the present model.

For the perturbation technique, we have initially assumed that we could split the given potential in two parts, Eq. (3). The main part corresponds to a shape invariant potential, Eq. (7), for which the superpotential is known analytically and the remaining part is treated as a perturbation, Eq. (8). If necessary, one can expand the functions related to the perturbation in terms of the perturbation parameter $\lambda$,

$$
\begin{aligned}
\Delta V(r ; \lambda) & =\sum_{k=1}^{\infty} \lambda^{k} \Delta V_{k}(r), \\
\Delta W_{n}(r ; \lambda) & =\sum_{k=1}^{\infty} \lambda^{k} \Delta W_{n k}(r), \\
\Delta \epsilon_{n}(\lambda) & =\sum_{k=1}^{\infty} \lambda^{k} \epsilon_{n k},
\end{aligned}
$$

where $\lambda$ will eventually be set equal to one. Substitution of the above expansion into Eq. (8) by equating terms with the same power of $\lambda$ on both sides yields up to $O\left(\lambda^{3}\right)$

$$
2 W_{n} \Delta W_{n 1}-\frac{\hbar}{\sqrt{2 m}} \Delta W_{n 1}^{\prime}=\Delta V_{1}-\Delta \epsilon_{n 1}
$$




$$
\begin{gathered}
\Delta W_{n 1}^{2}+2 W_{n} \Delta W_{n 2}-\frac{\hbar}{\sqrt{2 m}} \Delta W_{n 2}^{\prime}=\Delta V_{2}-\Delta \epsilon_{n 2}, \\
2\left(W_{n} \Delta W_{n 3}+\Delta W_{n 1} \Delta W_{n 2}\right)-\frac{\hbar}{\sqrt{2 m}} \Delta W_{n 3}^{\prime}=\Delta V_{3}-\Delta \epsilon_{n 3},
\end{gathered}
$$

which are exactly SSPT expressions appeared in [15]-[18] but for the case $n=0$. Eq. (8) and its expansion, Eqs. (11-13), give a flexibility for the easy calculations of the perturbative corrections to energy and wave functions for the $n^{\text {th }}$ state of interest through an appropriately chosen perturbed superpotential, unlike the other perturbation theories. We will show in the next section through the applications that this feature of the present model leads to a simple framework in obtaining the corrections to all states without using complicated and tedious mathematical procedures.

\section{Application}

\section{1 perturbed harmonic oscillator}

Let us start with the most elementary kind of perturbation calculation. Consider a perturbed harmonic oscillator potential of the form

$$
V(r ; \lambda)=\left[\frac{1}{2} m w^{2} r^{2}+\frac{\ell(\ell+1) \hbar^{2}}{2 m r^{2}}\right]+\frac{1}{2} m \lambda w^{2} r^{2},
$$

which woud arise by increasing the spring constant of a harmonic oscillator from

$K$ to $(1+\lambda) K$ since $w=\sqrt{K / m}$. The perturbed potential in its present form can of course be solved exactly. Neverthless, we want to solve this problem in the light of our approach to test the effectiveness of the present technique. This application will also clarify that our derivation is much simpler and more direct than the other methods, and hence provides a useful alternative.

The whole spectrum and corresponding wave functions for the unperturbed part of the problem is well known in the literature. Then, starting with the normalized wave function of the harmonic oscillator system for any state of interest, one can easily calculate the corrections at one step to the energy and eigenvalues for the perturbed potential setting an appropriate superpotential satisfying Eq. (8). For the comparison of our results with those of recent works, we will perform the calculations here for the ground state $n=0$. Starting with Eq. (8),

$$
\Delta W_{n=0}^{2}-\frac{\hbar}{\sqrt{2 m}} \Delta W_{n=0}^{\prime}+2 W_{n=0} \Delta W_{n=0}=\frac{1}{2} m \lambda w^{2} r^{2}-\Delta \epsilon_{n=0},
$$

one readily sees that the perturbed superpotential $(\Delta W)$ satisfying the above equation has the form

$$
\Delta W_{n=0}=\sqrt{\frac{m}{2}} w r(\sqrt{1+\lambda}-1),
$$


since, from the literature [11], the superpotential $W$ corresponding to the unperturbed potential is in the form

$$
W_{n=0}=\sqrt{\frac{m}{2}} w r-\frac{(\ell+1) \hbar}{\sqrt{2 m} r} .
$$

Upon substituting (16) and (17) into (15) yields

$$
\Delta \epsilon_{n=0}=\left(\ell+\frac{3}{2}\right)(\sqrt{1+\lambda}-1) \hbar w
$$

and using (16) with (2) leads to the moderating function for the ground state

$$
\phi_{n=0}=\exp \left[\frac{m w}{2 \hbar} r^{2}(1-\sqrt{1+\lambda})\right] .
$$

As the ground state wave function $\chi_{n=0}$ corresponding to the superpotential in (17) and energy $\epsilon_{n=0}$ for the normalized unperturbed harmonic oscillator are

$$
\chi_{n=0}=N r^{\ell+1} \exp \left(-\frac{m w r^{2}}{2 \hbar}\right), \epsilon_{n=0}=\left(\ell+\frac{3}{2}\right) \hbar w,
$$

the total wave function $\psi_{n=0}$ for the whole system in (14) and corresponding energy are obtained as

$\psi_{n=0}=\chi_{n=0} \phi_{n=0}=N r^{\ell+1} \exp \left(-\frac{m w \sqrt{1+\lambda}}{2 \hbar} r^{2}\right), E_{n=0}=\sqrt{1+\lambda}\left(\ell+\frac{3}{2}\right) \hbar w$.

These are indeed the correct results which can be verified explicitly by the use of Eq. (9), since this problem has an exact analytical solution.

Lee $[16,17]$ recently has studied this problem within the frame of supersymmetric perturbation theory and worked out it up to the second order. From the expansion of the closed expressions in (21) in $\lambda$ such that $\sqrt{1+\lambda}=1+\lambda / 2-$ $\lambda^{2} / 8+\ldots$, it can clearly be seen that Lee's treatment indeed appears as a subset of the present calculation procedure. In addition, our sophisticated approach neither involves cumbersome procedures nor tedious calculations. In what follows, we further show that the present model is also applicable in the same form to bound excited states without any difficulty, unlike the works in [15]-[17].

As the potential considered in (14) is a shape invariant potential, using (9) together with (16) and (17) for $n=0$ and having in mind the shape invariance property for the exactly solvable potentials [11],

$$
V_{+}\left(r, a_{0}\right)=V_{-}\left(r, a_{1}\right)+R\left(a_{1}\right) ; R\left(a_{1}\right)=2 \hbar w \sqrt{1+\lambda},
$$

where $V_{-}$is the exactly solvable potential in (9) and $V_{+}$is its supersymmetric partner

$$
\left(W_{n=0}+\Delta W_{n=0}\right)^{2}+\frac{\hbar}{\sqrt{2 m}}\left(W_{n=0}+\Delta W_{n=0}\right)^{\prime}=V_{+}-E_{n=0}
$$


and $a_{0}=\ell, a_{1}=\ell+1$. Therefore,

$$
E_{n}=\sum_{s=1}^{n} R\left(a_{s}\right)+\left(\ell+\frac{3}{2}\right) \hbar w=\left(2 n+\ell+\frac{3}{2}\right) \hbar w \sqrt{1+\lambda}
$$

which are the whole spectrum for the perturbed harmonic potential in (14). This very simple procedure removes difficulties encountered in SSPT and LPT in dealing with excited states. Again, this result agrees with Eq. (47) of [16] in which the excited state energies are presented up to only second order due to nasty calculation procedure.

To find the energy of the excited states, all we had to do was to perform calculations using a proper superpotential corresponding to the ground state wave function of the perturbed potential. The LPT literature goes to great pains to find ways to avoid using excited state wave functions. The study of present example, in particular the procedure used through Eqs. (22) to (24), thus illustrates in this respect how SSQM allows us to use the LPT based formulas for the entire spectrum, by moving to a partner potential whose ground state coincides with the excited state of the potential initillay considered. The reader is referred to [11] for a further discussion on supersymmetric partner potentials and their applications.

\section{2 perturbed Coulomb interaction}

Kim and Sukhatme [9] obtained a set of expressions for ground and excited state wave functions and energies in perturbation theory (henceforth referred to as $\mathrm{KS}$ ), that do not involve infinite sums, and which they consider a generalization of LPT. Subsequently some expressions in this formalism were simplified by Mavromatis [19]. Later, KS approach were carefully compared [20] with the results of the LPT formalism and step by step connection between the two formalisms was shown and commented on via an illustrative example, together with the discussion on the relation between KS approach to Dalgarno-Lewis formalism [5]-[8].

As we are aware of the relation between LPT and SSPT, one then may expect also the connection between SSPT and LPT based KS approach. Thus, we focus through this section and the other examples in the next sections on constructing a bridge between the present generalized supersymmetric perturbation formalism and KS approach and show explicitly the equivalence between the two aproaches, considering of course the expanded form of Eq. (8).

We proceed first with the present treatment of the simple example used in [20], which is the perturbed Coulomb system,

$$
V(r)=\left[-\frac{e^{2}}{r}+\frac{\ell(\ell+1) \hbar^{2}}{2 m r^{2}}\right]+\lambda \frac{e^{2}}{2 r},
$$

where, for a particular example, $\lambda$ denotes the increse in the charge of the nucleus in case of an interaction between electron and nucleus in hydrogen like atoms. 
The superpotential, wave function and energy for the ground state corresponding to the unperturbed potential, respectively, are [11]

$$
\begin{aligned}
W_{n=0} & =\sqrt{\frac{m}{2}} \frac{e^{2}}{(\ell+1) \hbar}-\frac{(\ell+1) \hbar}{\sqrt{2 m} r}, \\
\chi_{n=0} & =N r^{\ell+1} \exp \left(-\frac{m e^{2}}{(\ell+1) \hbar^{2}} r\right), \\
\epsilon_{n=0} & =-\frac{m e^{4}}{2 \hbar^{2}(\ell+1)^{2}} .
\end{aligned}
$$

From Eq. (8), $\Delta W_{n=0}$ involving all the corrections corresponding to the perturbing potential $\Delta V=\lambda e^{2} / 2 r$ is readily obtained as

$$
\Delta W_{n=0}=-\sqrt{\frac{m}{8}} \frac{\lambda e^{2}}{(\ell+1) \hbar}
$$

and the the total correction to the enery $\Delta \epsilon_{n=0}$, together with the moderating function due to the perturbing potential are

$$
\Delta \epsilon_{n=0}=-\frac{m e^{4}}{2 \hbar^{2}}\left(\frac{\lambda^{2}}{4}-\lambda\right) \quad, \quad \phi_{n=0}=\exp \left(\frac{\lambda m e^{2}}{2 \hbar(\ell+1)} r\right)
$$

Hence, the full ground state wave function and energy spectrum for the perturbed Coulomb system in (25) are

$$
\begin{aligned}
\psi_{n=0}=\chi_{n=0} \phi_{n=0} & =N r^{\ell+1} \exp \left[-\frac{m e^{2}}{(\ell+1) \hbar^{2}}\left(1-\frac{\lambda}{2}\right) r\right], \\
E_{n=0} & =-\frac{m e^{4}}{2 \hbar^{2}(\ell+1)^{2}}\left(1-\frac{\lambda}{2}\right)^{2} .
\end{aligned}
$$

One can justify the result above via Eq. (9) and the full excited energy spectrum can be easily found using the procedure given through Eqs (22-24), for which the superpotential should have the final form $W_{n=0}+\Delta W_{n=0}$.

Now, to see the close relation between the formalism presented in this letter and that of Kim and Sukhatme [9], the reader should go back to Eqs. (11-13) which are the expansion of the present formalism yielding individual corrections in order, having in mind that $\phi_{n}^{K S}$ is expanded in orders of $\lambda$ in KS approach. In the light of recent works $[16,17]$ regarding SSPT, one sees after some algebra that the expansion terms of moderating function $\phi_{n}^{K S}$ in KS technique are related to those of the superpotential $\Delta W_{n}$ appearing in (11) through (13). For clarity consider only the first order, then

$$
\Delta W_{n 1}=-\frac{\hbar}{\sqrt{2 m}} \frac{d}{d r} \phi_{n 1}^{K S}
$$


since, from (11),

$$
\Delta W_{n 1}(r)=\frac{\sqrt{2 m}}{\hbar} \frac{1}{\chi_{n}^{2}(r)} \int_{-\infty}^{r} \chi_{n}^{2}(z)\left[\Delta \epsilon_{n 1}-\Delta V_{1}(z)\right] d z .
$$

Mavromatis [20], using KS and LPT formalism and working within $\hbar=m=$ $e^{2}=1$ unit system for $\ell=0$ case, investigated the same problem for the ground state solution $(n=0)$ for which after some tedious integrals he arrived at

$$
\phi_{01}^{K S}=\frac{r-a}{2} \lambda, \quad \phi_{02}^{K S}=\frac{(r-a)^{2}}{8} \lambda^{2}, \quad \phi_{03}^{K S}=\frac{(r-a)^{3}}{48} \lambda^{3}, \ldots
$$

and $\Delta \epsilon_{01}=\lambda / 2, \Delta \epsilon_{02}=-\lambda^{2} / 8$ with higher order energy corrections being zero. We should remark at this point that the present approach has provided exact result in a closed analytical form, Eq. (28), without dealing with nasty integrals, since the moderating function in (32) eventually can be written as $\phi_{n}^{K S}=\exp [\lambda / 2(r-a)]$ with an extra constant, $\exp (-\lambda a / 2)$, though the constants do not affect the energy, where $a$ comes from the lower limit of the integrals carried out. Considering Eqs. $(30,32)$ within the same frame and the whole discussion given in this section, together with the physics behind Eqs. (8-13), one can see that the model introduced here through Eq. (8) unifies not only SSPT and LPT but also the KS approach. In the following section, we will make more clear this point with the further examples used in [9].

\section{3 harmonic oscillator with linear perturbation $(n=1$ state $)$}

Kim and his co-worker considered a one dimensional perturbed harmonic oscillator potential with no angular momentum barrier

$$
V=\frac{1}{4} w^{2} r^{2}+\lambda r+B
$$

involving a linear perturbation term. Picking the particular case of an applied uniform electric field to a charged particle moving in a simple harmonic oscillator potential provides a simple physical interpretation to the linear term in (33) where in this case $\lambda$ stands for the strenght of the field applied. This explanation is also valid for the next example considered in the following section but for a different unperturbed potential.

In [9], the energy level shifts and corrections to the wave function were worked out up to the second order for the first excited state $(n=1)$,

$$
\Delta \epsilon_{11}=B \quad, \quad \Delta \epsilon_{12}=-\frac{\lambda^{2}}{w^{2}}, \quad \phi_{11}^{K S}=-\frac{\lambda}{w}\left(r-\frac{2}{w r}\right), \quad \phi_{12}^{K S}=\frac{\lambda^{2} r^{2}}{2 w^{2}} .
$$


For a clear comparison we work within $\hbar=2 m=1$ unit system as in [9]. From (30) and (34), $\Delta W$ in the first order should read

$$
\Delta W_{11}=\frac{\lambda}{w}\left(1+\frac{2}{w r^{2}}\right),
$$

and the use of (35) in (11) yields explicitly the first order energy shift $\Delta \epsilon_{11}=B$, since

$$
\chi_{n=1}=N r \exp \left(-w r^{2} / 4\right) \quad, \quad W_{n=1}=\frac{w r}{2}-\frac{1}{r}, \epsilon_{n=1}=3 w / 2 .
$$

The second order calculations can be carried out in the same manner for comparison. However, our aim here is to clarify the effectiveness of the technique introduced in this article by calculating all corrections explicitly in a simple way. For this reason we turn back our attention to the shifted harmonic oscillator potential in (33) in order to remind the reader that the potential in (33) can be exactly solved in its present form by setting the superpotential

$$
W_{n=1}^{\text {exact }}=\frac{w r}{2}+\frac{\lambda}{w}
$$

from which one sees that $B=-w$ and $E_{n=1}^{\text {exact }}=3 w / 2+B-\lambda^{2} / w^{2}=\epsilon_{n=1}+$ $\Delta \epsilon_{11}+\Delta \epsilon_{12}$ which justifies the results obtained above. This makes clear that the energy level shifts due to higher orders in $\lambda$ vanish. Eq. (37) leads to the full wave function in the light of Eq. (6)

$$
\psi_{n=1}^{\text {exact }}=N_{1} \exp \left(-w r^{2} / 4\right) \exp (-\lambda r / w) .
$$

Further, it is not difficult to see that Eq. (9) provides the exact full energy spectrum involving all excited states using the shape invariance property of the potential in (33) without carrying out tedious integrals as in the other perturbation theories. The physics behind this exact solution would shed a light to be able to use Eq. (8) for an easy calculation of the contributions comes from due to the perturbed terms, which are briefly discussed below.

To illustrate once more the power and elegancy of the present formalism we here show that all the corrections to the sytem of interest can be calculated simply by Eq. (8). For the choice of correct superpotential leading to the linear perturbation term we use

$$
\Delta W_{n=1}=W_{n=1}^{\text {exact }}-W_{n=1}=\frac{\lambda}{w}+\frac{1}{r}
$$

and upon substituting (39) into (8) one clearly sees that full energy corrections are $\Delta \epsilon_{n=1}=\Delta \epsilon_{11}+\Delta \epsilon_{12}=B-\lambda^{2} / w^{2}$, together with the corresponding moderator function involving all the modifications

$$
\phi_{n=1}=\frac{\exp (-\lambda r / w)}{r} .
$$


Hence, the first excited state wave function for (33) reads

$$
\psi_{n=1}=\chi_{n=1} \phi_{n=1}=N \exp \left(-w r^{2} / 4\right) \exp (-\lambda r / w),
$$

from which it is clear that the present formalism produces exact results. However, the correction terms in KS approach [9] obtained for $n=1$ state up to the second order given in (34) deviate slightly from the exact result (see Eq. (25) of [9]) as in the previous example, while it leads to the correct answer for the correction terms to the energy.

\section{4 infinite square well with linear perturbation $(\mathrm{n}=2$ state $)$}

Finally, Kim and Sukhatme used their technique to investigate the $n=2$ state of an infinite square well potential

$$
V(r)= \begin{cases}0 & \text { if }|r| \leq \pi / 2 \\ \infty & \text { otherwise }\end{cases}
$$

subject to a perturbation $\Delta V(r)=\lambda r+B$. To calculate the energy level shifts and corrections to the unperturbed wave function

$$
\chi_{n=2}=\sqrt{\frac{2}{\pi}} \cos 3 r
$$

one first needs to define the corresponding superpotential

$$
W_{n=2}=-\frac{\chi_{n=2}^{\prime}}{\chi_{n=2}}=3 \tan 3 r .
$$

To see once more the close relation between KS approach and the present formalism, consider Eq. (11) in which $\Delta W_{21}$ can be obtained through (30)

$$
\Delta W_{21}=\frac{\lambda \sec ^{2} 3 r}{4}\left(\frac{\pi^{2}}{4}-r^{2}\right)-\frac{\lambda}{6}\left(r \tan 3 r+\frac{1}{6}\right),
$$

since the second order correction to the wave function $\phi_{21}^{K S}$ in [9] appears in the form

$$
\phi_{21}^{K S}=\frac{\lambda}{4}\left[\frac{1}{3}\left(r^{2}-\frac{\pi^{2}}{4}\right) \tan 3 r+\frac{1}{9} r\right] .
$$

Eq. (45) can also be checked out using (31) by setting the limits of the integral from $-\pi / 2$ to $\pi / 2$. Substitution of Eqs. (44) and (45) into (11) verifies the result $\Delta \epsilon_{21}=B$ in [9]. Similarly, the second order energy level correction found in [9] is

$$
\Delta \epsilon_{22}=\frac{\lambda^{2}}{36}\left(\frac{\pi^{2}}{12}-\frac{5}{36}\right)
$$


which can in our theoretical framework be obtained by

$$
\Delta \epsilon_{22}=-\int_{-\pi / 2}^{\pi / 2} \frac{2}{\pi} \cos ^{2} 3 r \Delta W_{21}^{2}(r) d r,
$$

that is derived from (12).

Again, the study of this example justifies that the formalism introduced in this letter for perturbative problems has a more general form, since the whole KS approach for the calculation of corrections due to perturbative terms is hidden in the subset of our formalism, namely the expansion of Eq. (8) leading to Eqs. (11) and (12) for the first and second order modifications reproduces same results as in KS formalism. Additionally, the mathematical treatment is much simpler in the frame of the present approach.

\section{Concluding Remarks}

No single approximation method available in the literature is ideal for every problem. SSPT and LPT based theories avoids the Rayleigh-Schrödinger summation, but it can lead to nasty integrals and more effort in particular for excited states. The method is valuable when the integrals can be done exactly or by a reliable numerical procedure. Otherwise, the Rayleigh-Schrödinger summation, even when it does not give an exact answer, starts not to look so bad after all. This was the motivation behind the work introduced in this article. The present perturbation model appears in this respect to be superior for the excited states and provides a quick route to the calculation of all corrections within the frame of the perturbation theory, which considerably simplify one's calculational workload.

Further, an attempt is made through this article to generalize SSPT and shown that the new formalism unifies SSPT- and LPT-based perturbation theories, which resolves difficulties and inefficiecies calculating in particular excited state corrections without having used tedious procedures. The power and elegancy of the unified model which is, in a sense, complete are illustrated via specifally chosen four examples. We now have clear and explicit ways to get corrections to all energy levels and state wave functions for a given perturbed potential.

As a concluding remark, SSQM has so far illuminated so many different areas of quantum mechanics that one might wonder what light perturbation theories based on supersymmetry might shed on the conventional techniques. In that hope, we are not about to be disappointed. We believe that this simple and intuitive method discussed through this paper would find a widespread application in the related area. The application of the method to other potentials involving Yukawa and Woods-Saxon potentials is in progress. 


\section{References}

[1] Aharonov Y. and Au C. K. 1979 Phys. Rev. Lett. 421582.

[2] Au C. K. and Aharonov Y. 1979 Phys. Rev. A 2022.

[3] Imbo T. and Sukhatme U. P. 1984 Am. J. Phys. 52140.

[4] Au C. K., Chan K. L., Chow C. K., Chu C. S. and Young K 1991 J. Phys. A: Math. Gen. 243837.

[5] Dalgarno A. and Lewis J. T. 1955 Proc. R. Soc. 23370.

[6] Schwartz C. 1959 Ann. Phys. 2156.

[7] Schwartz C. 1959 Ann. Phys. 2170.

[8] Mavromatis H. A. 1991 Am. J. Phys. 59738.

[9] Kim I. W. and Sukhatme U. P. 1992 J. Phys. A: Math. Gen. 25 L647.

[10] Dobrovolska I. V. and Tutik R. S. 1999 J. Phys. A: Math. Gen. 32563.

[11] Cooper F., Khare A. and Sukhatme U. P. 1995 Phys. Rep. 251267.

[12] Junker G. 1996 Supersymmetric Methods in Quantum and Statistical Physics, Springer, Berlin.

[13] Nicolai H. 1976 J. Phys. A: Math. Gen. 91497.

[14] Witten E. 1981 Nucl. Phys. B 188513.

[15] Cooper F. and Roy P. 1990 Phys. Lett. A 143202.

[16] Lee C. 2000 Phys. Lett. A 267101.

[17] Lee C. 1999 senior thesis Reed collage.

[18] Chakrabarti B. and Das T. K. 2001 Phys. Lett. A 28511.

[19] Mavromatis H. A. 1993 J. Phys. A: Math. Gen. 26 L515.

[20] Mavromatis H. A. 1996 Tr. J. Phys. 201202. 